\title{
Papers
}

\section{Deep vein thrombosis and air travel: record linkage study}

\author{
C W Kelman, M A Kortt, N G Becker, Z Li, J D Mathews, C S Guest, C D J Holman
}

\begin{abstract}
Objective To investigate the time relations between long haul air travel and venous thromboembolism.

Design Record linkage study using the case crossover approach.

Setting Western Australia.

Participants 5408 patients admitted to hospital with venous thromboembolism and matched with data for arrivals of international flights during 1981-99.

Results The risk of venous thromboembolism is increased for only two weeks after a long haul flight; 46 Australian citizens and 200 non-Australian citizens had an episode of venous thromboembolism during this so called hazard period. The relative risk during this period for Australian citizens was 4.17 (95\% confidence interval, 2.94 to 5.40 ), with $76 \%$ of cases $(\mathrm{n}=35)$ attributable to the preceding flight. A "healthy traveller" effect was observed, particularly for

Australian citizens.

Conclusions The annual risk of venous thromboembolism is increased by $12 \%$ if one long haul flight is taken yearly. The average risk of death from flight related venous thromboembolism is small compared with that from motor vehicle crashes and injuries at work. The individual risk of death from flight related venous thromboembolism for people with certain pre-existing medical conditions is, however, likely to be greater than the average risk of 1 per 2 million for passengers arriving from a flight. Airlines and health authorities should continue to advise passengers on how to minimise risk.
\end{abstract}

\section{Introduction}

Venous thromboembolism after air travel was first recorded in 1954, but the magnitude of risk has not been resolved. ${ }^{12}$ An increase in the risk of thrombosis in the legs of passengers undertaking long haul flights could be caused by long term immobilisation in cramped seating, low atmospheric pressure, or dehydration, acting in synergy with personal risk factors (see bmj.com). ${ }^{2}{ }^{3}$ Recent small studies have shown relative risks of between 1 and 4 for venous thromboembolism occurring two to four weeks after a flight, the so called hazard period. ${ }^{45}$ The incidence of pulmonary embolism among 135 million passengers arriving at Charles de Gaulle airport was much greater among those who had travelled more than $10000 \mathrm{~km}$ (4.8 per million arrivals); flight duration seems to be a critical trigger. ${ }^{26} \mathrm{~A}$ few widely publicised deaths from pulmonary embolism after long flights has brought the issue to public attention.

The incidence of venous thromboembolism ranges from 1000-2000 per million person years for deep vein thrombosis and 500-1000 per million person years for pulmonary embolism. The incidence of venous thromboembolism increases with age. Around 1-2\% of venous thromboembolic events result in death. ${ }^{78}$ The post-thrombotic syndrome-a long term disabling consequence of deep vein thrombosis-develops in $10-30 \%$ of patients with venous thromboembolism. ${ }^{9}$

International air travel has increased to around 1.56 billion person trips each year. ${ }^{10}$ At any one time an estimated 4000 Australians are on international flights, and more than 30000 make short domestic flights each day.

Since 1970, Australia has kept electronic data on arrivals and departures of international travellers. The state of Western Australia uses record linkage under well developed protocols to protect patient privacy. ${ }^{11}$ Most Western Australian residents live in Perth, and flight times from there to other major airports are long. We investigated the relation between international air travel and venous thromboembolism by linking Western Australian hospital data with records on air travel.

\section{Participants and methods}

Data included coded personal identifiers, age, sex, arrival and departure dates, and nationality of the traveller (Australian citizen or non-Australian citizen). Non-Australian citizens included temporary residents, visitors, and a few people with permanent resident status. We used hospital discharge summaries for the period 1981-99 to detect all patients in Western Australia who had been admitted with a principal diagnosis of deep vein thrombosis or pulmonary embolism. These data were used to estimate age and sex specific incidences for venous thromboembolism and expected numbers of venous thromboembolic events, using state-wide incidence.

The travel records for all passenger movements to and from Australia during 1981-99 were probabilistically matched to the Western Australian hospital data for the same period. ${ }^{12}$ We thus identified patients with venous thromboembolism who had undertaken international flights, and we recorded the date of patients' first admission for venous thromboembolism and flight dates.
Commonwealth Department of Health and Ageing, GPO Box 9848 , Canberra, ACT 2601, Australia C W Kelman medical adviser M A Kortt assistant director J D Mathews deputy chief medical officer

National Centre for Epidemiology and Population Health, Australian National University,

Canberra, ACT

0200

N G Becker

professor of

biostatistics

$\mathrm{Z} \mathrm{Li}$

postdoctoral fellow

C S Guest

visiting fellow

School of

Population Health,

University of

Western Australia,

Perth, WA 6009,

Australia

C D J Holman

chair in public health

Correspondence to:

C W Kelman

christopher.kelman@ health.gov.au

bmj.com 2003;327:1072

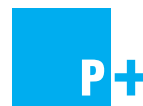

Risk factors for venous

thromboembolism appear on bmi.com 


\section{Bias}

Travellers who had acute venous thromboembolism after leaving Australia would not be admitted to hospital in Western Australia, and so that episode would not be captured in our data. We therefore used information on passengers arriving only. The diagnosis of venous thromboembolism was unlikely to be biased by a recent flight being mentioned during consultation, as there was little awareness of flight related venous thromboembolism before 1999 .

Information on travel and venous thromboembolism is incomplete while travellers are outside Western Australia. It was assumed that most non-travel time for Australian citizens resident in Western Australia would be spent in Western Australia. In contrast, many flights and hospital admissions for travellers not usually resident in Western Australia would not be visible in the data, as many international visitors leave Western Australia within days or weeks of arrival. Thus the denominator for observed number of non-Australian citizens with venous thromboembolism immediately after arrival is larger than for later after arrival. This results in proportionally more patients with venous thromboembolism being detected soon after arrival. To minimise this potential bias, our main analysis is based on Australian citizens, with summary statistics for non-Australian citizens.

Finally, venous thromboembolism in Western Australian citizens would not be captured in our data if it led to admission to hospital immediately after arrival from an overseas flight at an east coast airport and before proceeding to Perth. Such an effect would seem to be small (see figure).

A few patients with venous thromboembolism could have been missed owing to failure to make a link or missing data. The matching process was, however, set to maximise specificity, and such errors would underestimate any association. Data quality may have improved between 1981 and 1999, and by the late 1990s deep vein thrombosis was more likely to be treated on an outpatient basis with anticoagulants, in accordance with new treatment protocols. ${ }^{13}$ No time related bias was found in sensitivity analyses by using different time periods.

\section{Statistical analysis}

Our principal analysis looked for evidence of any temporary increase in risk of venous thromboembolism above baseline after arrival from a flight. The method uses the conditional probability distribution of the time from the start of the observation period until the first venous thromboembolic event, given that this event occurred within the observation period. This approach is similar to a case crossover analysis. ${ }^{14}{ }^{15}$ Because the incidence is low, the risk was estimated as an incidence rate ratio, which gives an approximate relative risk. The incident rate ratio during the hazard period, relative to periods beyond the immediate post flight period was modelled as a function of age, sex, and time since flight. The appendix describes the analysis in detail. This formulation gives the same estimate as the method of Marshall and Jackson, when their approach uses an individual's entire flight history to estimate the probability of a flight at any point in time..$^{15}$

\section{Hazard period and sensitivity analyses}

The estimated incident rate ratio is influenced by the duration of the post flight period selected for analysis. A 30 day period of increased risk has been assumed previously, and a recent study reported a median interval of two days (range 1-14) between a trigger event and subsequent thrombosis. ${ }^{16}$ We examined the risk over intervals up to 30 days, using a model that allowed different incident rate ratios in three successive periods after travel, and we concluded that most of the excess risk was captured in the two weeks after a flight.

We analysed data subsets for 1981-89, 1981-99, 1990-5, and 1990-9. As these estimates were consistent, we present those for 1981-99, which exploits all available data.

\section{Results}

During 1981-99, 4.8 million Australian citizens and 4.6 million non-Australian citizens arrived in Western Australia from an international flight. Over the same period there were 16205 hospital admissions (13 184 patients) for venous thromboembolism diagnosed by usual clinical criteria in Western Australian hospitals; $84 \%(\mathrm{n}=11075)$ of patients had one admission for venous thromboembolism $(44 \% ; n=4844)$ had pulmonary embolism diagnosed. When linked to travel data, these 13184 patients were found to include 2279 (17\%) Australian citizens and 3129 (24\%) nonAustralian citizens with a record of an international flight arrival during the study period. Table 1 summarises their characteristics.

\section{Hazard period and risk for Australian citizens}

We identified 153 Australian citizens who were admitted to hospital for venous thromboembolism within 100 days of arrival on an international flight. The figure shows the time between arrival and first admission for a venous thromboembolic event for these 153 Australian citizens; 46 events occurred within 14 days of arrival, and 107 events occurred 15-100 days after arrival. The observed number of cases within 14 days of arrival significantly exceeds the number expected under the assumption of a uniform distribution over the $0-100$ days after arrival $\left(\chi^{2}=30.3\right.$, $\mathrm{df}=1, \mathrm{P}<0.001)$. Table 2 presents the observed and expected numbers of all patients admitted to hospital within the first 100 days of arrival. The observed numbers for Australian citizens are less than those expected from Western Australian population rates, suggesting a "healthy traveller" effect.

Table 1 Characteristics of patients with venous thromboembolism with history of preceding international flight. Values are means (standard deviations) unless stated otherwise

\begin{tabular}{lccccc} 
Nationality & No & Age at arrival & $\begin{array}{c}\text { Duration of hospital } \\
\text { stay }\end{array}$ & Death rate (\%) & Frequency (SD) of flight \\
\hline Australian citizens & 2279 & $53(17)$ & $7.4(6.6)$ & 2.2 & $2.4(2.9)$ \\
\hline Non-Australian citizens & 3129 & $56(16)$ & $7.6(6)$ & 2.1 & $4.3(4.9)$ \\
\hline
\end{tabular}

${ }^{\star}$ Average over study period. 


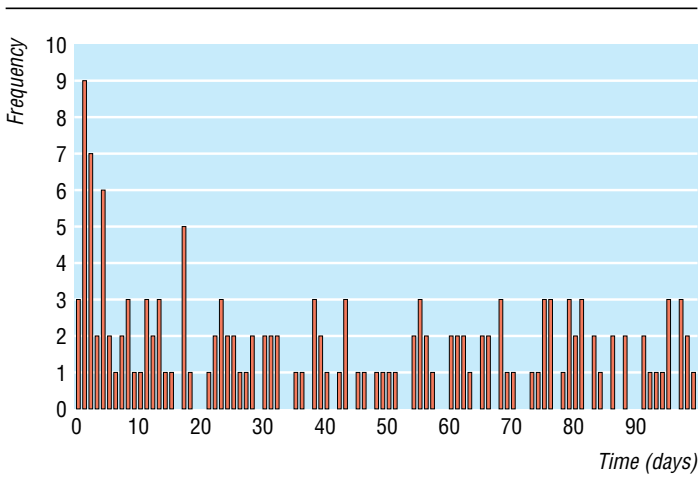

Frequency of venous thromboembolism in Australian citizens $(n=153)$ by days after flight arrival for first 100 days. Day 0 was counted as 0.5 days

The maximum likelihood analysis showed that the incidence rate ratio was increased for Australian citizens within two weeks of arrival, falling from 5.61 (95\% confidence interval, 3.94 to 7.97$)$ in week 1 to 2.63 (1.55 to 4.45 ) in week 2 (table 3 ). The increase did not depend significantly on age or sex, and it was not significant in week 3 (see models in table 3). The data are not consistent with a constant hazard rate over the first two weeks (likelihood ratio test: $\chi^{2}=6.05, \mathrm{df}=1$, $\mathrm{P}=0.014)$. To estimate the relative risk within two weeks of arrival, we used the pooled (average) incident rate ratio from the parsimonious model $([7.5 \times 5.61+7 \times 2.63] / 14.5=4.17 ; 95 \%$ confidence interval, 2.94 to 5.40). From the relative risk of 4.17 in the hazard period, the attributable fraction for a patient having venous thromboembolism within two weeks of arrival was $76 \%$-that is, $3.17 / 4$.17. Table 4 gives details of those admitted to hospital within two weeks of arrival. Assuming one long haul trip per year, the additional risk due to this exposure amounts to an extra two weeks at $(4.17-1)=3.17$, which corresponds to $3.17 \times 2 / 52=12 \%$ extra risk over the entire year.

\section{Observations in non-Australian citizens and mortality}

Overall, 200 non-Australian citizens with venous thromboembolism were detected within two weeks of arrival. This significantly exceeds the 105 expected on the basis of Western Australian population rates $\left(\chi^{2}=85.2, \mathrm{df}=1, \mathrm{P}<0.001\right)$. As the exact distribution of length of stay for non-Australian citizens in Western Australia is unknown, it was not possible to completely separate the declining rate for venous thromboembolism over time from bias, owing to the declining denominator for non-Australian citizens over time. We therefore do not show the incident rate ratios for nonAustralian citizens.

Five of the 246 patients with venous thromboembolism detected within the hazard period died in hospital. Because our study was based on patients admitted to hospital we could have missed a few cases presenting as sudden death either in flight or soon after arrival.

\section{Discussion}

Venous thromboembolism is four times more likely to develop within two weeks of arrival from a long haul flight, the so called hazard period. Although we tried to minimise the effects of possible biases in our study, our results should be generalised with caution. It would be convenient to assume that, outside the hazard period, an air traveller has the same risk of venous thromboembolism as the average for people of the same age and sex in Western Australia. However, this assumption leads to an expectation of around 600 patients with venous thromboembolism among Australian citizens within 15-100 days of arrival, compared with an observed number of only 107 . This suggests a

Table 2 Observed and expected numbers of first venous thromboembolism events by time in days since most recent flight arrival

\begin{tabular}{|c|c|c|c|c|c|}
\hline \multirow[b]{2}{*}{ Traveller category } & \multicolumn{5}{|c|}{ Days since most recent flight arrival } \\
\hline & $0-14^{\star}$ & 15-30 & $31-60$ & $61-100$ & Total \\
\hline \multicolumn{6}{|l|}{ Australian citizens: } \\
\hline Observed & 46 & 23 & 32 & 52 & 153 \\
\hline Expected $(A) \dagger$ & 102.6 & 113.2 & 212.3 & 283.0 & 711.1 \\
\hline Expected $(B) \ddagger$ & 22.1 & 24.4 & 45.7 & 60.9 & 153.0 \\
\hline \multicolumn{6}{|l|}{ Non-citizens: } \\
\hline Observed & 200 & 69 & 78 & 91 & 438 \\
\hline Expected $(\mathrm{A}) \S$ & 105.3 & 116.2 & 217.9 & 290.5 & 729.9 \\
\hline Expected (B) & 63.2 & 69.7 & 130.7 & 174.3 & 438.0 \\
\hline
\end{tabular}

${ }^{*} \chi^{2}=30.3, \mathrm{df}=1, \mathrm{P}<0.001$ when patients with venous thromboembolism in first 14 days are compared with expected (B).

tCalculated from total number of arrivals of Australian citizens in Western Australia (4.8 million) and using age and sex specific rates for venous thromboembolism in Western Australian population. These expectations illustrate scale of "healthy traveller" effect.

¥Calculated on assumption that 153 observed venous thromboembolism events of first 100 days are uniformly distributed in time (for example, $153 \times 14.5 /$

$100.5=22.1$ ). For non-citizens in $0-14$ day interval, expectation $(A)$ is biased upwards if anything, whereas expectation (B) is biased downwards because of early

departures of visitors from Western Australia. Expectations for citizens are likely to be more robust.

$\S \chi^{2}=85.2, \mathrm{df}=1, \mathrm{P}<0.001$ when patients with venous thromboembolism in first 14 days are compared with expected (A).

Table 3 Estimates (95\% confidence intervals) of incidence rate ratios for Australian citizens arriving from overseas and known to have venous thromboembolism (1981-99)

\begin{tabular}{lccccc} 
Model type & $\exp \left(\boldsymbol{\beta}_{1}\right)(\text { days } \mathbf{0}-7)^{\star}$ & $\exp \left(\boldsymbol{\beta}_{\mathbf{2}}\right)($ days $\mathbf{8}-\mathbf{1 4})$ & $\exp \left(\boldsymbol{\beta}_{3}\right)($ days $\mathbf{1 5}-\mathbf{2 1})$ & $\exp \left(\boldsymbol{\beta}_{\mathbf{s}}\right)(\boldsymbol{s e x})$ & $\exp \left(\boldsymbol{\beta}_{\mathbf{a}}\right)(\mathbf{a g e})$ \\
\hline Full & $5.17(3.21$ to 8.31$)$ & $2.42(1.31$ to 4.49$)$ & $1.38 \dagger(0.64$ to 2.98$)$ & $1.18 \dagger(0.68$ to 2.06$)$ & $1.01 \dagger(0.99$ to 1.02$)$ \\
\hline Parsimonious & $5.61(3.94$ to 7.97$)$ & $2.63(1.55$ to 4.45$)$ & - & - & - \\
\hline
\end{tabular}

${ }^{\star}$ First week is 7.5 days as time to event is calculated from day 0.5 .

†Variables testing for age and sex dependence of incidence rate ratio and for excess risk in third week are not statistically significant. Variables $\left(\beta_{3}, \beta_{\mathrm{s}}, \beta_{\mathrm{a}}\right)$ are dropped from final (parsimonious) model. 
healthy traveller effect. Those who undertake international travel may be more healthy and thus less likely to develop spontaneous venous thromboembolism than those who do not travel, presumably because people with risk factors are less likely to fly (see bmj.com).

\section{Absolute risk owing to international air travel}

Of 4.8 million Australian citizens arriving in Western Australia during 1981-99, 46 developed venous thromboembolism within the hazard period. This corresponds to an absolute rate of around 9.6 patients with venous thromboembolism per million people arriving, of which 7.3 per million would be attributable to the preceding flight. Of around 4.6 million non-Australian citizens arriving in Western Australia, there were 200 patients with venous thromboembolism in the hazard period, with an estimated 33 cases per million people arriving attributable to the preceding flight (data not shown). This higher estimate for the absolute risk for non-Australian citizens is not due to differences in age and sex, as the distributions for arrivals of Australian and non-Australian citizens were similar. The greater risk in non-Australian citizens could be due to longer flights from Europe, in contrast to the generally shorter flights of Western Australian residents, who more often visit South East Asia. ${ }^{6}$ The study data did not, however, allow us to test the effect of flight duration on the risk of venous thromboembolism. It is also possible that non-Australian citizens with an incentive to visit Western Australia are less healthy on average than Australian citizens who choose to leave Western Australia for tourist or business purposes. Other selective biases could apply-for example, the threshold for hospital admission in Australian citizens with a family doctor could be higher than for visitors, who might present directly to a hospital emergency department.

We found a $12 \%$ increase in annual risk of venous thromboembolism for a traveller undertaking one long flight yearly. However, as citizens who travel seem more healthy than non-travellers, the absolute risk for an average traveller will be less than that estimated from

Table 4 Characteristics of 246 travellers (Australian citizens and non-Australian citizens) admitted to hospital with venous thromboembolism within 14 days of arrival from an international flight for study period 1981-99

\begin{tabular}{lccc} 
Age group & $\begin{array}{c}\text { No of patients with venous } \\
\text { thromboembolism }\end{array}$ & $\begin{array}{c}\text { Total No of people } \\
\text { arriving }\end{array}$ & $\begin{array}{c}\text { Rate per } \mathbf{1 0 0} \text { o00 people } \\
\text { arriving }^{*}\end{array}$ \\
\hline $0-14$ & 0 & 1118070 & 0.00 \\
\hline $15-19$ & 2 & 834355 & 0.24 \\
\hline $20-24$ & 4 & 950621 & 0.42 \\
\hline $25-29$ & 4 & 909289 & 0.44 \\
\hline $30-34$ & 4 & 910372 & 0.44 \\
\hline $35-39$ & 7 & 924062 & 0.76 \\
\hline $40-44$ & 22 & 870695 & 2.53 \\
\hline $45-49$ & 31 & 717674 & 4.32 \\
\hline $50-54$ & 40 & 570198 & 7.02 \\
\hline $55-59$ & 25 & 308846 & 8.09 \\
\hline $60-64$ & 30 & 457647 & 6.56 \\
\hline $65-69$ & 29 & 336319 & 8.62 \\
\hline $70-74$ & 27 & 200015 & 13.50 \\
\hline$\geq 75$ & 21 & 149679 & 14.03 \\
\hline All & 246 & 9257842 & 2.66
\end{tabular}

140 patients had deep vein thrombosis and 106 had pulmonary embolism. Five deaths occurred in hospital. * Rates are reasonable approximation as only $16 \%$ of patients had more than one venous thromboembolism event and it would be expected that few would have had multiple flight related events.

\section{What is already known on this topic}

Venous thromboembolism has been suggested to be up to four times more likely to develop within two to four weeks of a flight (the "hazard period")

The incidence of pulmonary embolism is greater among passengers travelling more than $10000 \mathrm{~km}$

\section{What this study adds}

The risk of venous thromboembolism is highest within two weeks of a long haul flight

The annual risk of venous thromboembolism is increased by $12 \%$ in those undertaking one long haul flight a year

rates in a population that includes non-travellers as well as travellers. If venous thromboembolism risks apparently caused by air travel to Western Australia are extrapolated nationally, around 27 venous thromboembolism episodes per million people arriving would be expected, or about 250 episodes in 2003. Most of these episodes would be in non-Australian citizens. Australian citizens would probably be at similar risk from flights leaving Australia (with venous thromboembolic events occurring in other countries). We found that around five deaths per year from pulmonary embolism could be attributable to international flights terminating in Australia, which would correspond to a death rate of 1 per 2 million people arriving. These results are generally consistent with other reports. $^{4-6} 1718$

\section{Comparative risks}

Various types of unintentional injuries are responsible for around 260 deaths per million person years in Australia. ${ }^{19}$ Deaths from an incident on leading international airlines occur at a rate of about 0.1 per million person years, which is close to the risk of death from lightning strike. ${ }^{20}$ The rate for deaths from motor vehicle crashes in Australia is about 100 per million person years. ${ }^{21}$ Thus for an average international traveller, the annual risk of death in a motor vehicle incident would be around 100 times greater than the additional risk of death from pulmonary embolism from a return flight. Even people who fly frequently (an international flight every fortnight) would be more likely to die on the roads. Thus the additional risk to the individual is small compared with other generally accepted risks.

Although the average risk of venous thromboembolism is small, prospective passengers with certain medical conditions are likely to have a higher than average risk of flight related venous thromboembolism because of the underlying risk (see bmj.com). Airlines and health authorities should continue to advise passengers on ways to minimise that risk. Further studies of predisposing factors and causal mechanisms are needed, as are evaluations of the preventive measures already introduced by airlines and health authorities. ${ }^{22}$ Future research should also define the healthy traveller effect and better quantify risk in relation to duration of travel. 
We thank Diana Rosman (Data Linkage Unit, Western Australia), Bill McLoughlin, Greg McNeilly, and Terry Cooper (Department of Immigration, Multicultural and Indigenous Affairs) for their assistance in extracting the data, Jim Manning, Antonia Lehn, and Jill Chorazy (Department of Transport and Regional Services) for their support, Rob Wooding, Philip Clarke, James Jordan, and Richard Smallwood (Commonwealth Department of Health and Ageing), and Dorothy Broom (Australian National University) for invaluable input.

Contributors: CWK, JDM, CSG, and CDJH conceived the study. Data analysis was performed by CWK, MAK, JDM, NGB, and ZL. CWK, MAK, NGB, JDM, CSG, and CDJH drafted the paper. JDM will act as guarantor for the paper.

Funding: Commonwealth Department of Transport and Regional Services. ZL was supported by the National Health and Medical Research Council grant No 148919. The guarantor accepts full responsibility for the conduct of the study, had access to the data, and controlled the decision to publish.

Competing interests: None declared.

Ethical approval: The research protocol was approved by the ethics committee of the Commonwealth Department of Health and Ageing, the human research ethics committee at the University of Western Australia, and the Confidentiality of Health Information Committee of the Western Australian Department of Health.

\section{Appendix: method of analysis adapted to study objective and type of data}

The sample consists of individuals who experienced at least one venous thromboembolic event during the time interval from the start of 1981 until the end of 1999. Consider a time interval $[0, \tau]$, which may be the entire interval or a subset thereof. Suppose that the hazard rate for the onset of a venous thromboembolic event for an individual labelled $j$ is $\lambda_{\mathrm{j}}(\mathrm{t})$ at time $t$ of this time interval. This rate might depend on characteristics of the individual, some of which are observed and others not. The characteristic of primary interest here is the individual's travel history, which is available for each case over this time interval.

Beginning at time 0 , consider the time $T_{j}$ until individual $j$ has the first venous thromboembolic event. For any time $t$ in interval $[0, \tau]$, we have $\operatorname{Pr}(T>t)=$ $\exp \left[-\Lambda_{j}(t)\right]$, where:

\section{$\Lambda_{j}(t)=\int_{0}^{t} \lambda_{j}(u) d u$, the area under the curve $\lambda_{i}(t)$.}

The nature of our sampling ensures that the first onset occurs in $[0, \tau]$. We therefore need the conditional probability distribution, given this event, which is:

$$
\operatorname{Pr}\left(T_{j} \leq t \mid T_{j}<\tau\right)=\left\{1-\exp \left[-\Lambda_{j}(t)\right]\right\} /\left\{1-\exp \left[-\Lambda_{j}(\tau)\right]\right\} \approx
$$
$\Lambda_{j}(t) / \Lambda_{j}(\tau)$,

where the last approximation holds because $\Lambda_{\mathrm{j}}(\tau)$ is small.

For individual $j$ we observe $T_{j}=t_{j}$. The contribution to the likelihood from this observation is:

\section{(1) $\lambda_{j}\left(t_{j}\right) / \Lambda_{j}(\tau)$}

In contrast to the corresponding expression in the case crossover analysis of Marshall and Jackson, the integral in the denominator of (1) is over time rather than over the covariates. ${ }^{15}$

The aim is to allow the hazard rate to be altered during a time interval immediately after each long flight. To add flexibility we divided the post flight interval into three parts, with the first of duration $y_{1}$, the second of duration $\mathrm{y}_{2}$, and the third of duration $\mathrm{y}_{2}$. After any flight, the hazard rate is assumed to be $b_{1 j} \lambda_{0 j}$ during the first post flight period, $b_{2 j} \lambda_{0 j}$ during the second post flight period, $b_{3 j} \lambda_{0 \mathrm{j}}$ during the third post flight period, and $\lambda_{0 \mathrm{j}}$ otherwise. Letting the $\lambda_{0 \mathrm{j}}$ differ between individuals allows individuals to differ in their background or baseline predisposition to venous thromboembolism. When we assume that $\lambda_{0 j}$ does not depend on time it cancels out of the likelihood contribution (1), and this is where the analogy with the case crossover design comes in. Rate ratios $b_{1 j}, b_{2 i}$, and $b_{3 j}$ larger than 1 correspond to increased hazard rates in the three short periods after a flight. It is of interest to see if the amount of increase, if any, depends on other characteristics of the individual. Here we allow for sex and age:

$$
b_{i j}=\exp \left[\beta_{i}+\beta_{s} \cdot \operatorname{sex}_{j}+\beta_{A}\left(a_{0 j}-\bar{a}_{0}\right)\right]
$$

where $\operatorname{sex}_{j}$ is 0 if individual $j$ is male and 1 if female, whereas $\mathrm{a}_{0 \mathrm{j}}$ is the age of individual $j$ at time 0 and $\overline{\mathrm{a}}_{0}$ is the sample average age at time 0 . The likelihood function is given by the product of the contributions (1) over all individuals in the sample. Then maximum likelihood estimates can be obtained for the variables $\beta_{1}, \beta_{2}$, $\beta_{3}, \beta_{\mathrm{s}}$, and $\beta_{\mathrm{a}}$, including $95 \%$ confidence intervals based on the normality of maximum likelihood estimates from large samples. The likelihood estimating equations were solved by Newton-Raphson iteration.

Homans J. Thrombosis of the leg veins due to prolonged sitting. N EnglJ Med 1954;250:148-9.

Ansell JE. Air travel and venous thromboembolism-is the evidence in? N Engl J Med 2001;345:828-9.

3 Gallus AS, Baker RI. Economy class syndrome. Med I Aust 2001;174:264-5

4 Kraaijenhagen RA, Haverkamp D, Koopman MM, Prandoni P, Piovella F, Buller HR. Travel and risk of venous thrombosis. Lancet 2000;356:1492-3.

5 Ferrari E, Chevallier T, Chapelier A, Baudouy M. Travel as a risk factor for venous thromboembolic disease: a case-control study. Chest 1999:115:440-4

6 Lapostolle F, Surget V, Borron SW, Desmaizieres M, Sordelet D, Lapandry $\mathrm{C}$, et al. Severe pulmonary embolism associated with air travel. $N$ Engl J Med 2001;345:779-83.

7 Rosendaal FR. Venous thrombosis: a multicausal disease. Lancet 1999;353:1167-73.

8 Anderson FA, Wheeler HB, Goldberg RJ, Hosmer DW, Patwardhan NA, Jovanovic B, et al. A population based perspective of the hospital incidence and case fatality rates of deep vein thrombosis and pulmonary embolism. The Worcester DVT study. Arch Intern Med 1991;151:933-8.

9 Prandoni P, Lensing AWA, Cogo A, Cuppini S, Villalta S, Carta M, et al. The long-term course of acute deep venous thrombosis. Ann Intern Med The long-term

10 International Civil Aviation Organisation. 1999 Annual report of the council:International civil aviation organisation. Montreal, Quebec: ICAO; 2000. 11 Holman CD, Bass AJ, Rouse IL, Hobbs MS. Population-based linkage of health records in Western Australia: development of a health services research linked database. Aust NZ J Public Health 1999;23:453-9.

12 Kelman CW, Bass AJ, Holman CD. Research use of linked health data-a best practice protocol. Aust NZ J Public Health 2002;26:251-5.

13 Levine M, Gent M, Hirsch J, Leclerc J, Anderson D, Weitz J, et al. A comparison of low-molecular-weight heparin administered primarily at home with unfractionated heparin administered in the hospital for home with unfractionated heparin administered in the hospit
proximal deep-vein thrombosis. N Engl J Med 1996;334:677-81.

14 Maxlure M. The case-crossover design: a method for studying transient effects on the risk of acute events. Am J Epidemiol 1991;133:144-53.
ent

15 Marshall RJ, Jackson RT. Analysis of case-crossover designs. Stat Med 1993;12:2333-41.

16 Eekhoff EM, Rosendaal FR, Vandenbroucke JP. Minor events and the risk of deep vein thrombosis. Thromb Haemost 2000;83:408-11.

17 Kesteven PJ, Robinson BJ. Clinical risk factors for venous thrombosis associated with air travel. Aviat Space Environ Med 2001;72:125-8.

18 Lord RSA. Air travel-related venous thromboembolism, Sydney views. Hawaii Med J 2000;59:155-6.

19 Australian Bureau of Statistics. 1998 causes of death. Canberra: ABS, 1999.

20 National Transportation Safety Board. Accidents, fatalities, and rates, 1982 through 2001, for U.S. air carriers operating under 14 CFR 121, scheduled through 2001, for U.S. air carriers operating und
service (airlines). Washington DC: NTSB, 2001.

21 Department of Transport and Regional Development. Road fatalities Australia: 1998 statistical summary. Canberra: DTRD, 1999.

22 Scurr JH, Machin SJ, Bailey-King S, Mackie IJ, McDonald S, Smith PD. Frequency and prevention of symptomless deep-vein thrombosis in long-haul flights: a randomised trial. Lancet 2001;357:1485-9.

(Accepted 15 August 2003) 